\title{
Corrigendum to: Mendelian disorders of the epigenetic machinery: postnatal malleability and therapeutic prospects
}

Jill A. Fahrner ${ }^{1,2}$, Hans T. Bjornsson ${ }^{1,2,3,4, *}$

${ }^{1}$ McKusick-Nathans Institute of Genetic Medicine, 21205 2Department of Pediatrics, Johns Hopkins University School of Medicine, Baltimore, MD 21205, USA ${ }^{3}$ Landspitali University Hospital, Reykjavik 101, Iceland and ${ }^{4}$ Faculty of Medicine, University of Iceland, Reykjavik 101, Iceland

*To whom correspondence should be addressed at: Johns Hopkins University, School of Medicine, 733 N. Broadway, MRB 415, MD 21205, USA. Tel: 4105020056; Fax: 4106149246. Email: hbjorns1@jhmi.edu

Human Molecular Genetics, 2019, 28(R2), R254-R264.

doi: $10.1093 / \mathrm{hmg} / \mathrm{ddz} 174$.

"Kabuki syndrome 2" was added in error to the table in association with the gene "KMT2D" after the authors submitted the final proof. The press apologizes for this error. 